\title{
Preditores de Desempenho Escolar no 5을 Ano do Ensino Fundamental
}

\author{
Edna Maria Marturano \\ Universidade de São Paulo \\ Ribeirão Preto, SP, Brasil \\ Elaine Cristina Gardinal Pizato \\ Fundação Educacional Dr. Raul Bauab \\ Jaú, SP, Brasil
}

\begin{abstract}
RESUMO
Diferenças individuais de desempenho podem ser atribuídas a características do aluno, da família e da escola. O objetivo deste estudo prospectivo foi testar um modelo de predição de desempenho no $5^{\circ}$ ano do ensino fundamental $-\mathrm{EF}$, tendo como preditores habilidades acadêmicas e sociais, problemas de comportamento e percepção de estressores escolares no $3^{\circ}$ ano, bem como o tempo de exposição à educação infantil - EI e a condição socioeconômica do alunado da escola de EF. Participaram 248 alunos de quatro escolas públicas. Os instrumentos utilizados foram: Critério de Classificação Econômica Brasil, Teste de Desempenho Escolar, Social Skills Rating System - SSRS versão do professor, Inventário de Estressores Escolares e avaliação coletiva de desempenho. Habilidades acadêmicas, habilidades sociais e percepção de stress no $3^{\mathrm{O}}$ ano predisseram desempenho no $5^{\mathrm{O}}$ ano. A escola de EF afetou diferentemente a avaliação objetiva de desempenho e o julgamento de competência acadêmica feito pelo professor.
\end{abstract}

Palavras-chave: Desempenho acadêmico; Problemas de comportamento; Educação infantil; Gênero; Ensino fundamental.

\section{ABSTRACT}

\section{Predictors of Academic Achievement in the 5th Grade of Elementary School}

Student, family and school factors account for individual differences in school performance through elementary school (EF). The aim of this prospective study was to test a model to predict academic performance in the 5th year of EF, adopting academic skills, social skills, behavior problems and perceived school hassles in the third year as predictors, as well as the length of exposure to early childhood education - ECE and the socioeconomic background of the student population in each EF school. Participants were 248 students attending four public schools. The instruments used were: Brazil Economic Classification Criteria, Academic Performance Test, teacher Social Skills Rating System - SSRS, Inventory of School Hassles and a collective assessment of school performance. Academic skills, social skills, and perceived stress in the third year predicted further academic performance. EF school effects on objective performance assessment were different from their effects on the teacher's judgment of academic competence.

Keywords: Academic performance; Behavior problems; Early childhood education; Gender; School.

\section{RESUMEN}

\section{Predictores de Rendimiento Escolar en el Quinto Año de la Escuela Primaria}

Diferencias individuales de rendimiento se pueden atribuir al alumno, a la familia y a la escuela. El objetivo del estudio fue testar un modelo predictivo de rendimiento en el quinto año de la escuela primaria - EP, teniendo como predictores habilidades académicas y sociales, problemas de comportamiento y el estrés percibido en el tercer año, así como el tiempo en la educación preescolar y la situación socioeconómica de los estudiantes de la EP. Participaron 248 estudiantes de cuatro escuelas públicas. Los instrumentos fueron: Criterio de Clasificación Económica Brasil, Prueba de Rendimiento Académico, Social Skills Rating System - SSRS versión del profesor, Inventario de Estrés Escolar, evaluación colectiva de desempeño. Habilidades académicas, habilidades sociales y el estrés percibido en el tercer año predijejon el rendimiento en el quinto. La situación socioeconómica de la EP ha afectado de manera diferente el desempeño en la prueba objectiva y la competencia evaluada por el profesor.

Palabras clave: Rendimiento escolar; Problemas de comportamiento; Educación preescolar; Género; Escuela primaria. 
O aprendizado escolar é multideterminado. Tendo como pano de fundo a influência bem documentada do nível socioeconômico - NSE, diferenças individuais de desempenho acadêmico no ensino fundamental EF podem ser tributadas a características do aluno, da família, da escola e até mesmo da vizinhança, respondendo inclusive, quando combinadas, pela associação entre NSE e resultados escolares (Aikens \& Barbarin, 2008). Neste artigo, focalizam-se características do aluno associadas à influência da escola, na predição de resultados acadêmicos.

Pesquisas com foco nas características do aluno mostram que o desempenho escolar correlaciona positivamente com habilidades sociais e negativamente com problemas de comportamento, seja na categoria de externalização, que inclui agressão e violação de regras, seja na de internalização, que traduz ansiedade, humor deprimido, retraimento, queixas somáticas (Ackerman, Izard, Kobak, Brown, \& Smith., 2007; Chen, Huang, Chang, Wang, \& Dan, 2010; Konold, Jamison, StantonChapman, \& Rimm-Kaufman, 2010). A influência positiva das habilidades sociais sobre o desempenho parece bem estabelecida em estudos longitudinais entre o jardim de infância e o $3^{\circ}$ ano (Romano, Babchishin, Pagani, \& Kohen, 2010), a educação infantil e o $5^{\mathrm{o}}$ ano (Konold et al. , 2010) e o $3^{\circ}$ e o $8^{\circ}$ ano (Caprara, Barbaranelli, Pastorelli, Bandura, \& Zimbardo, 2000). Pesquisas sugerem influências transacionais, ou seja, habilidades sociais favorecem o futuro desempenho acadêmico e vice-versa (Chen et al., 2010).

A predição de desempenho acadêmico a partir de diferenças individuais em ajustamento comportamental apresenta resultados mistos. Grover, Ginsburg e Ialongo (2007) verificaram que crianças altamente ansiosas no $1^{\mathrm{O}}$ ano tinham desempenho prejudicado no $5^{\mathrm{O}}$ ano, ao passo que Ackerman et al. (2007) encontraram indícios de internalização das dificuldades acadêmicas entre o 3 e o 5ㅇa ano do EF. Burt e Roisman (2010) verificaram que comportamentos externalizantes avaliados pelas mães na pré-escola predizem desempenho no $1^{\mathrm{O}}$ ano do $\mathrm{EF}$ e o desempenho no $1^{\mathrm{O}}$ ano do $\mathrm{EF}$, por sua vez, prediz internalização no $3^{\circ}$ ano.

Os resultados das pesquisas sugerem influências recíprocas entre percursos de desempenho acadêmico, habilidades sociais e problemas de comportamento. Assim, parece ser importante, na predição do desempenho acadêmico em um momento dado da trajetória escolar da criança, levar em consideração os níveis prévios de habilidades sociais e problemas de comportamento, no mesmo modelo preditivo. Burt e Roisman (2010), adotando essa perspectiva, encontraram relações diversas daquelas verificadas nas pesquisas em que apenas habilidades sociais ou problemas de comportamento foram considerados. Nos seus resultados, não se confirmou a influência positiva das habilidades sociais sobre o desempenho acadêmico, demonstrada em outros estudos; em contrapartida, sobressaiu a influência dos comportamentos externalizantes precoces, tanto sobre o desempenho escolar como sobre a competência social avaliados em anos subsequentes, bem como efeitos destes sobre comportamentos internalizantes mais tardios.

A experiência subjetiva da criança na escola também pode afetar seu desempenho, sendo, por sua vez, influenciada por propriedades do contexto escolar. Sabe-se que as crianças percebem a escola como importante fonte de stress, particularmente em questões relacionadas ao desempenho (Byrne, Thomas, Burchell, Olive, \& Mirabito, 2011; Leung, Yeung, \& Wong, 2010). Também há evidência de associação entre a percepção de maior stress na escola e um desempenho escolar mais fraco (Marturano, TrivellatoFerreira, \& Gardinal, 2009).

A percepção de estressores acadêmicos parece estar associada à pressão por bons resultados escolares (Byrne et al., 2011) e pode contribuir para sintomas internalizantes. (Leung, Yeung, \& Wong, 2010). No estudo de Byrne et al. (2011), a percepção de estressores acadêmicos no EF correlacionou com ansiedade avaliada 24 meses depois. Tais efeitos podem contribuir para a internalização das dificuldades acadêmicas, como observado por Ackerman et al.(2007). Pode-se então supor que a percepção de estressores relacionados ao desempenho tenha um papel na predição do desempenho futuro, em processo de retroalimentação.

A permanência na escola contribui efetivamente para o desenvolvimento das crianças. A passagem pela Educação Infantil - EI tem efeitos acadêmicos, cognitivos e psicossociais positivos (Felício, Terra, \& Zoghbi, 2012; Tagartt, Sylva, Melhuish, Sammons, \& Siraj-Blatchford, 2011). No EF também há evidência de que a escola contribui para o progresso do aluno (Rutter \& Maugham, 2002). No entanto, crianças que frequentam escolas diferentes não têm chances iguais de progresso acadêmico, haja visto que mesmo entre escolas da rede pública de um único município podem ser encontradas diferenças de desempenho no alunado (Salles, Parente, \& Freitas, 2010).

Além das condições intrínsecas à escola, como a qualidade do ensino, a organização, o clima e a segurança (Rutter \& Maugham, 2002), condições extrínsecas como a origem social dos alunos afetam o desempenho individual. A concentração de alunos em desvantagem socioeconômica é condição de vulnerabilidade para problemas de desempenho e 
comportamento (Aikens \& Barbarin, 2008; Rutter \& Maugham, 2002).

Com base no exposto a respeito de influências do aluno e da escola sobre o desempenho, parece relevante investigar tais efeitos em modelo que contemple tanto variáveis do aluno como da escola. No entanto, faltam na literatura empírica tentativas de integração das informações esparsas disponíveis, de modo a estimar o peso relativo de cada variável relevante na predição do progresso acadêmico, bem como investigar como cada um desses preditores se comporta em presença dos demais. Contribuições têm sido feitas no sentido de modelar influências conjuntas de diferentes contextos (ver, por exemplo, Aikens e Barbarin, 2008) ou explorar efeitos transacionais entre variáveis do aluno (Burt \& Roisman, 2010; Chen et al., 2010). Os resultados promissores de tais iniciativas autorizam supor que seria igualmente produtivo explorar efeitos conjuntos de variáveis do aluno e do contexto. Com o presente estudo se pretende dar um passo nessa direção. Seu objetivo é testar um modelo de predição de desempenho acadêmico no $5^{\circ}$ ano do $\mathrm{EF}$, tendo como preditores do aluno as habilidades acadêmicas e sociais, os problemas de comportamento e a percepção de estressores acadêmicos no $3^{\circ}$ ano, e como preditores escolares o tempo de exposição à EI e características sociodemográficas da escola de EF. Controlam-se efeitos de NSE e gênero, fatores influentes no desenvolvimento do escolar (Aikens \& Barbarin, 2008; Konold et al., 2010).

\section{MÉTODO}

\section{Participantes}

Participaram 248 alunos do 3을 ano do EF em quatro escolas públicas de uma cidade do Estado de São Paulo. As escolas obtiveram, no Índice de Desenvolvimento da Educação Básica - IDEB, avaliação superior à média do Estado em 2009. Foi critério de inclusão o aluno ter idade de 7 a 9 anos e pelo menos um ano completo na EI. Compuseram a amostra 125 meninos e 132 meninas, com idade média de 8,43 anos (desviopadrão 0,34 ), sendo que 74 tinham feito um ano de EI e 174 tinham completado dois anos. Mães e professores participaram como informantes.

\section{Instrumentos}

Para avaliar o NSE foi utilizado o Critério de Classificação Econômica Brasil (Associação Brasileira de Empresas de Pesquisa, 2003). A caracterização das escolas de EF foi baseada em três indicadores: mobilidade dos alunos durante a coleta (mudança de cidade), NSE médio dos alunos do $3^{\circ}$ ano $(\mathrm{N}=357)$ e porcentagem de alunos do $3^{\mathrm{o}}$ ano sem acesso prévio à EI.

As habilidades acadêmicas básicas no $3^{3}$ ano foram avaliadas com o Teste de Desempenho Escolar - TDE (Stein, 1994). O desempenho acadêmico no $5^{\mathrm{O}}$ ano foi avaliado por meio de dois instrumentos. Um deles foi a avaliação coletiva de português e matemática elaborada por Escolano (2004), que relata correlação de 0,78 entre a pontuação obtida nessa prova e a avaliação realizada pela escola na $4^{\mathrm{a}}$ série (atual $5^{\circ}$ ano). O outro foi a Escala de Competência Acadêmica do Sistema de Avaliação de Habilidades Sociais -- SSRS-BR, versão do professor (Bandeira, Del Prette, Del Prette, \& Magalhães, 2009). As habilidades sociais, os comportamentos externalizantes e os comportamentos internalizantes também foram avaliados pelo professor com o SSRS-BR. A percepção da criança sobre estressores acadêmicos foi avaliada pelo Fator 1 do Inventário de Estressores Escolares, com itens como "meus pais queriam que eu tirasse notas boas" e "a professora falou que eu tenho que melhorar" (Gardinal-Pizato, 2010; Marturano, Trivellato-Ferreira, \& Gardinal, 2009).

\section{Procedimento}

A pesquisa atende às diretrizes da Resolução 196/96 do Conselho Nacional de Saúde do Ministério da Saúde. O projeto foi aprovado por Comitê de Ética em Pesquisa. Os pais e os professores foram informados sobre os objetivos, os procedimentos e a livre participação. Somente participaram da pesquisa crianças cujos pais assinaram o Termo de Consentimento Livre e Esclarecido e que mediante consulta verbal aceitaram participar.

A coleta de dados ocorreu em duas ondas. No $3^{\text {o }}$ ano do EF cada criança participou de uma sessão individual, em que foram aplicados o TDE e o Inventário de Estressores Escolares. O SRSS-BR foi preenchido pelos professores. O formulário do Critério de Classificação Econômica Brasil foi preenchido pelos pais ou responsáveis durante reuniões de pais promovidas nas escolas. No $5^{\mathrm{O}}$ ano do ensino fundamental, vinte a 22 meses depois da primeira coleta, repetiu-se o procedimento com os professores; as crianças participaram da Prova de Avaliação Coletiva nas salas de aula, com a presença do professor da turma.

$\mathrm{Na}$ análise dos dados, verificou-se inicialmente a adequação das distribuições para o emprego de testes paramétricos, com base nas medidas de curtose e simetria. As distribuições apresentaram valores de curtose entre 2,6 e -1,7 e valores de simetria entre 1,5 e $-1,4$, possibilitando o uso de análises paramétricas. 
Precedendo as análises de regressão, foram exploradas as associações entre variáveis. Diferenças entre médias foram analisadas, por meio do teste $t$ de Student ou análise de variância, tendo como fatores de segmentação o gênero, o tempo de exposição à EI e a escola de EF. As variáveis da criança, avaliadas no $3^{\circ}$ e no $5^{\circ}$ ano, foram correlacionadas entre elas e com NSE por meio do coeficiente $r$ de Pearson.

Nas análises de regressão, o modelo de predição testou a contribuição relativa das variáveis da criança no $3^{\mathrm{o}}$ ano para os desfechos observados no $5^{\mathrm{o}}$ ano, controladas as variáveis gênero $(0=$ masculino, $1=$ feminino $)$ e NSE $(5=$ classe A, $1=$ classe $\mathrm{E})$. Foram processadas duas regressões, uma para desempenho e a outra para competência acadêmica no $5^{\circ}$ ano. Como preditores, entraram no primeiro bloco as variáveis sociodemográficas (gênero e NSE), o tempo de permanência na EI e a classificação da escola de EF de acordo com as características da clientela. No segundo e no terceiro bloco foram inseridos, respectivamente, os indicadores de competência (desempenho no TDE e habilidades sociais) e dificuldades adaptativas (problemas de comportamento e percepção de stress), avaliados no $3^{\circ}$ ano. Utilizou-se um nível alfa de 0,05 .

\section{RESULTADOS}

A pontuação das escolas, na Tabela 1, foi consistente entre os três indicadores, permitindo ordená-las em quatro níveis (Escola Alfa, nível 1, resultados mais pobres; Escola Delta, nível 4, melhores resultados). Houve razoável concordância com a classificação das escolas no IDEB em 2009, apenas diferindo a posição relativa das escolas Alfa e Beta.
TABELA 1

Caracterização das escolas segundo indicadores da clientela

\begin{tabular}{|c|c|c|c|c|}
\hline \multirow{2}{*}{ Variável } & \multicolumn{4}{|c|}{ Escola } \\
\hline & Alfa & Beta & Gama & Delta \\
\hline $\begin{array}{l}\text { Mobilidade durante a } \\
\text { coleta } 3^{\circ} \text { ano }\end{array}$ & $19 \%$ & $19 \%$ & $14 \%$ & $6 \%$ \\
\hline $\begin{array}{l}\text { Alunos do } 3^{0} \text { ano sem } \\
\text { acesso prévio à EI }\end{array}$ & $17 \%$ & $15 \%$ & $4 \%$ & - \\
\hline $\begin{array}{l}\text { Nível socioeconômico } \\
\text { alunos 3ํano* }\end{array}$ & 11,65 & 11,86 & 15,69 & 16,03 \\
\hline
\end{tabular}

* média

As médias e desvios-padrão das variáveis da criança são apresentadas na Tabela 2, agrupadas por gênero, anos na EI e escolas de EF participantes do estudo. Na tabela também se encontram os valores de $\mathrm{F}$ e $\mathrm{t}$.

Foram detectadas diferenças de gênero. No $3^{\circ}$ ano, as meninas foram avaliadas com mais habilidades sociais $\left(\mathrm{t}_{246}=2,81, \mathrm{p}<0,01\right)$ e os meninos com mais comportamentos externalizantes $\left(\mathrm{t}_{246}=4,81, \mathrm{p}<0,0001\right)$ e internalizantes $\left(\mathrm{t}_{246}=3,20, \mathrm{p}<0,01\right)$. No $5^{\mathrm{o}}$ ano as meninas tiveram melhor desempenho $\left(\mathrm{t}_{246}=2,56\right.$, $\mathrm{p}<0,05)$ e competência acadêmica $\quad\left(\mathrm{t}_{246}=2,79\right.$, $\mathrm{p}<0,01$ ).

O tempo na EI foi associado a melhor desempenho na avaliação coletiva no $5^{\circ}$ ano $\left(t_{246}=2,22, p<0,05\right)$. A escola de EF foi associada a todos os resultados da criança, exceto os de competência acadêmica. Nas avaliações do $3^{\circ}$ ano, foram observadas diferenças entre as escolas no TDE $\left(\mathrm{F}_{3,244}=14,70, \mathrm{p}<0,0001\right)$, habilidades sociais $\left(\mathrm{F}_{3,244}=7,81, \mathrm{p}<0,0001\right)$, externalização $\left(\mathrm{F}_{3,244}=18,06, \mathrm{p}<0,0001\right)$, internalização $\left(\mathrm{F}_{3,244}=18,58\right.$, $\mathrm{p}<0,0001)$ e percepção de stress escolar $\left(\mathrm{F}_{3,244}=5,45\right.$, $\mathrm{p}<0,01)$. No $5^{\circ}$ ano, as diferenças incidiram sobre

TABELA 2

Médias e desvios-padrão das variáveis do estudo, por gênero, anos na EI e escola de EF

\begin{tabular}{|c|c|c|c|c|c|c|c|c|}
\hline \multirow{3}{*}{ Variáveis } & \multicolumn{2}{|c|}{ Gênero } & \multicolumn{2}{|c|}{ Anos na Educação Infantil } & \multicolumn{4}{|c|}{ Escola de Ensino Fundamental } \\
\hline & Meninos & Meninas & 1 & 2 & Alfa & Beta & Gama & Delta \\
\hline & $(N: 125)$ & $(N: 123)$ & $(N: 74)$ & $(N: 174)$ & $(N: 81)$ & $(N: 56)$ & $(N: 50)$ & $(N: 61)$ \\
\hline Desempenho TDE $3^{\circ} 3$ & $80,8(28,9)$ & $86,7(27,5)$ & $78,7(28,3)$ & $85,8(28,1)$ & $72,1(34,4)$ & $76,4(27,4)$ & $95,4(16,8)$ & $96,2(17,2)$ \\
\hline Habilidades sociais $3^{\circ}$ 1,3 & $46,5(14,0)$ & $51,1(12,0)$ & $48,1(10,9)$ & $49,0(14,1)$ & $46,6(11,4)$ & $46,3(13,0)$ & $46,7(12,2)$ & $55,5(14,4)$ \\
\hline Externalização $3^{\circ}$ 1,3 & $9,4(7,0)$ & $5,4(6,2)$ & $7,5(6,5)$ & $7,4(7,1)$ & $10,8(6,9)$ & $7,8(6,2)$ & $2,7(5,3)$ & $6,5(6,2)$ \\
\hline Internalização $3^{\mathrm{O}} 1,3$ & $4,2(3,3)$ & $3,3(2,8)$ & $3,7(3,2)$ & $3,5(3,1)$ & $5,1(2,6)$ & $2,4(2,4)$ & $1,7(2,2)$ & $4,2(3,7)$ \\
\hline Stress escolar $3^{\circ} 3$ & $10,6(7,7)$ & $9,0(7,1)$ & $9,1(7,1)$ & $10,1(7,6)$ & $11,5(7,3)$ & $11,4(8,5)$ & $7,2(7,2)$ & $6,0(0,8)$ \\
\hline $\begin{array}{l}\text { Desempenho avaliação } \\
\text { coletiva } 5^{\circ} 1,2,3\end{array}$ & $9,4(4,1)$ & $10,7(3,9)$ & $9,2(3,5)$ & $10,4(4,2)$ & $8,6(3,9)$ & $8,3(3,3)$ & $11,4(3,5)$ & $12,5(3,7)$ \\
\hline Competência acadêmica 5으 1 & $33,7(9,4)$ & $36,9(8,6)$ & $35,5(7,1)$ & $35,1(9,9)$ & $35,3(9,1)$ & $34,8(7,6)$ & $36,5(9,1)$ & $34,5(10,5)$ \\
\hline
\end{tabular}

Nota: diferenças estatisticamente significativas entre grupos, $\mathrm{p}<0,05:{ }^{1}$ para gênero; ${ }^{2}$ para anos na educação infantil; ${ }^{3}$ para escola. 
o desempenho na avaliação coletiva $\left(\mathrm{F}_{3,244}=20,33\right.$, $\mathrm{p}<0,0001)$. Testes post-hoc entre pares de escolas indicaram que as diferenças no geral favoreceram as escolas Gama e Delta; no entanto, a escola Delta, nível 4, mostrou média mais alta de externalização em relação à Escola Gama (nível 3) e de internalização em relação às escolas Beta (nível 2) e Gama.

As correlações entre as variáveis da criança e entre estas e o NSE são apresentadas na Tabela 3. As medidas de competência correlacionaram positivamente umas com as outras, assim como as medidas de dificuldade adaptativa. Inversamente, medidas de competência e de disfunção apresentaram correlações negativas entre elas. O NSE foi associado a todas as variáveis da criança, exceto a competência acadêmica avaliada pelo professor no $5^{\circ}$ ano.
Os resultados das análises de regressão estão na Tabela 4, com os modelos de predição de desfechos acadêmicos no $5^{\mathrm{o}}$ ano. No modelo preditivo de desempenho na avaliação coletiva, as variáveis do primeiro bloco respondem por $21 \%$ da variação nos resultados obtidos no $5^{\circ}$ ano. Gênero, NSE e a escola de EF são preditores significativos, com maior peso para a escola, segundo os valores de beta padronizados. Com a introdução, no bloco 2, dos recursos da criança avaliados no $3^{\circ}$ ano, o poder de predição do modelo se eleva para $62 \%$ do total da variância nos resultados. Tanto as habilidades acadêmicas como as habilidades sociais predizem o desfecho. Deve-se acrescentar que, dentre as variáveis do bloco 1 , apenas a escola de EF continua no modelo, com valor de beta padronizado reduzido de 0,31 para 0,10 .

TABELA 3

Correlações entre as variáveis avaliadas no $3^{\mathrm{O}}$ e no $5^{\mathrm{O}}$ ano do ensino fundamental

\begin{tabular}{|c|c|c|c|c|c|c|c|}
\hline Variáveis & 1 & 2 & 3 & 4 & 5 & 6 & 7 \\
\hline 1. Desempenho TDE $3^{\circ}$ ano & - & & & & & & \\
\hline 2. Habilidades sociais $3^{\circ}$ ano & $0,35 * *$ & - & & & & & \\
\hline 3. Externalização 3ำ ano & $-0,35^{* *}$ & $-0,49 * *$ & - & & & & \\
\hline 4. Internalização $3^{\circ}$ ano & $-0,30 * *$ & $-0,43^{* *}$ & $0,52 * *$ & - & & & \\
\hline 5. Stress escolar $3^{\circ}$ ano & $-0,40 * *$ & $-0,28 * *$ & $0,26 * *$ & $0,16^{*}$ & - & & \\
\hline 6. Desemp. av. coletiva $5^{\circ}$ ano & $0,73 * *$ & $0,48 * *$ & $-0,42 * *$ & $-0,35^{* *}$ & $-0,47 * *$ & - & \\
\hline 7. Competência acad. $5^{\circ}$ ano & $0,55^{* *}$ & $0,45 * *$ & $-0,32 * *$ & $-0,42 * *$ & $-0,38 * *$ & $0,63 * *$ & - \\
\hline 8. Nível socioeconômico & $0,26 * *$ & $0,20 * *$ & $-0,23 * *$ & $-0,17 * *$ & $-0,22 *$ & $0,33 * *$ & 0,12 \\
\hline
\end{tabular}

$\mathrm{N}=248 ; * \mathrm{p}<0,05 ; * \mathrm{p}<0.01$.

TABELA 4

Modelos de regressão hierárquica examinando preditores de desempenho acadêmico no $5^{\circ}$ ano, avaliados no $3^{\circ}$ ano

\begin{tabular}{|c|c|c|c|c|c|c|c|c|}
\hline \multirow{2}{*}{ Preditores } & \multicolumn{4}{|c|}{ Avaliação coletiva } & \multicolumn{4}{|c|}{ Competência acadêmica SSRS } \\
\hline & $B(S E)$ & $\beta$ & $R^{2}$ & $\Delta R^{2}$ & $B(S E)$ & $\beta$ & $R^{2}$ & $\Delta R^{2}$ \\
\hline \multicolumn{9}{|l|}{ Bloco 1} \\
\hline Gênero & $0,92(0,47)$ & $0,11^{*}$ & & & $3,01(1,15)$ & $0,16^{*}$ & & \\
\hline Nível socioeconômico & $0,79(0,28)$ & $0,18 *$ & & & $1,25(0,68)$ & 0,13 & & \\
\hline Anos na EI & $0,26(0,52)$ & 0,03 & & & $-0,71(1,29)$ & $-0,04$ & & \\
\hline Escola EF & $0,25(0,05)$ & $0,31 * *$ & & & $-0,09(0,13)$ & $-0,05$ & & \\
\hline $\mathrm{F}_{(4,243)}$ variação & \multicolumn{2}{|c|}{$15,74^{* *}$} & 0,21 & 0,21 & \multicolumn{2}{|c|}{$2,82 *$} & 0,04 & 0,04 \\
\hline \multicolumn{9}{|l|}{ Bloco 2} \\
\hline Desempenho TDE & $0,08(0,01)$ & $0,58 * *$ & & & $0,17(0,02)$ & $0,54 * *$ & & \\
\hline Habilidades sociais SSRS & $0,98(0,17)$ & $0,24 * *$ & & & $2,52(0,48)$ & $0,28 * *$ & & \\
\hline $\mathrm{F}_{(2,241)}$ variação & \multicolumn{2}{|c|}{$129,29 * *$} & 0,62 & 0,41 & \multicolumn{2}{|c|}{$85,49 * *$} & 0,44 & 0,40 \\
\hline \multicolumn{9}{|l|}{ Bloco 3} \\
\hline Externalização - SSRS & $-0,01(0,03)$ & $-0,02$ & & & $0,01(0,08)$ & 0,01 & & \\
\hline Internalização - SSRS & $-0,01(0,06)$ & $-0,01$ & & & $-0,63(0,1)$ & $-0,21 * *$ & & \\
\hline Stress escolar - IEE & $-0,08(0,02)$ & $-0,15^{* *}$ & & & $-0,1(0,06)$ & $-013 *$ & & \\
\hline $\mathrm{F}_{(3,238)}$ variação & \multicolumn{2}{|c|}{$4,32 *$} & 0,64 & 0,02 & \multicolumn{2}{|c|}{$8,69 * *$} & 0,49 & 0,05 \\
\hline
\end{tabular}

$\mathrm{N}=248$. 
O acréscimo, no bloco 3, das medidas de disfunção obtidas no $3^{\mathrm{o}}$ ano contribui com apenas $2 \%$ para a porcentagem de variação dos resultados da avaliação coletiva explicados pelo modelo. A percepção de stress escolar é o único preditor significativo nesse bloco. Além disso, a escola de EF não mais prediz o desfecho. O modelo final, que explica $64 \%$ das diferenças individuais na avaliação coletiva de desempenho realizada no $5^{\circ}$ ano, inclui, por ordem de importância, as seguintes variáveis da criança avaliadas no $3^{\circ}$ ano: habilidades acadêmicas básicas, habilidades sociais e percepção de stress (preditor negativo).

No modelo preditivo de competência acadêmica, avaliada pelo professor, as variáveis do primeiro bloco respondem por $4 \%$ da variação nos resultados obtidos no $5^{\circ}$ ano. Somente o gênero (se menina) aparece como preditor positivo de competência no $5^{\mathrm{o}}$ ano. Ao serem introduzidos na regressão os recursos da criança avaliados no $3^{\circ}$ ano (bloco 2), o modelo passa a explicar $44 \%$ do total da variância nos resultados do $5^{\circ}$ ano. Habilidades acadêmicas e sociais no $3^{\circ}$ ano são preditoras de competência acadêmica; o exame dos valores de beta padronizado indica que as habilidades acadêmicas são o melhor preditor isolado. Ao passo que o gênero deixa de ser um preditor significativo, a escola de EF passa a fazer parte do modelo, como um preditor negativo de competência acadêmica $(\beta=-0,25)$.

O acréscimo, no bloco 3, das medidas de disfunção obtidas no $3^{\circ}$ ano contribui com $5 \%$ para a porcentagem de variação dos resultados de competência acadêmica explicados pelo modelo. Sintomas de internalização e percepção de stress são os preditores significativos nesse bloco. O modelo final, que explica $49 \%$ das diferenças individuais em competência acadêmica no $5^{\mathrm{O}}$ ano, inclui, por ordem de importância, as seguintes variáveis avaliadas no $3^{\circ}$ ano: habilidades acadêmicas básicas, escola de EF (preditor negativo), habilidades sociais, sintomas de internalização (preditor negativo) e percepção de stress (preditor negativo).

\section{DISCUSSÃO}

O presente estudo teve como objetivo testar um modelo de predição de desempenho acadêmico no $5^{\mathrm{O}}$ ano do EF, tendo como preditores as habilidades acadêmicas e sociais, os problemas de comportamento e a percepção de estressores escolares no $3^{\text {o }}$ ano, considerando ainda a extensão do tempo de exposição à EI e características da clientela da escola de EF. Análises preliminares detectaram algumas associações previamente referidas na literatura, como um melhor desempenho acadêmico nas meninas (Aikens \&
Barbarin, 2008), nas crianças com maior tempo na EI (Tagartt et al., 2011; Felício et al., 2012) e nos alunos de escolas com clientela de origem social mais favorecida (Aikens \& Barbarin, 2008).

Variáveis da criança avaliadas no $3^{\circ}$ ano, quando correlacionadas a medidas de desempenho no $5^{\mathrm{O}}$ ano, também mostraram associação na direção esperada com base em pesquisas prévias. Confirmaram-se os achados de que, em algum grau, habilidades sociais mais desenvolvidas predizem melhor desempenho (Caprara et al., 2000; Chen et al., 2010; Konold et al., 2010; Romano et al., 2010), ao passo que um desempenho mais fraco é associado a dificuldades adaptativas prévias, seja no que diz respeito aos comportamentos externalizantes e internalizantes (Burt \& Roisman, 2010; Grover et al., 2007), seja no concernente à percepção de estressores relacionados ao desempenho (Marturano et al., 2009).

As análises de regressão por blocos, em modelo que segregou efeitos de preditores ambientais, recursos e dificuldades adaptativas do aluno, revelaram como esses preditores se comportam, quando considerados em conjunto e com o controle das variáveis NSE e gênero. $\mathrm{O}$ modelo explicou $64 \%$ da variância nos resultados de desempenho acadêmico, avaliado por uma prova objetiva aplicada coletivamente na sala de aula, e 49\% da variância nos resultados de competência acadêmica avaliada pelo professor. As habilidades acadêmicas no $3^{\mathrm{o}}$ ano foram o melhor preditor de desfechos no $5^{\mathrm{o}}$ ano, quer na avaliação objetiva de conteúdos escolares, quer na apreciação do professor. Romano et al. (2010) encontraram resultados semelhantes, em estudo prospectivo que investigou o efeito de habilidades de linguagem, aritmética, atenção e indicadores socioemocionais, avaliados no jardim de infância, sobre desempenho em leitura e aritmética no $3^{\circ}$ ano. Os resultados do presente estudo estendem esses achados, em um cenário que leva em conta o tempo de exposição à EI e características socioeconômicas do alunado da escola de EF.

As habilidades sociais predisseram desempenho e competência acadêmica no $5^{\mathrm{O}}$ ano, reiterando achados prévios de efeitos positivos das habilidades sociais no progresso escolar durante o EF (Caprara et al., 2000; Konold et al., 2010; Chen et al., 2010). Em conjunto, habilidades sociais e acadêmicas avaliadas no $3^{\circ}$ ano respondem por $41 \%$ e $40 \%$ da variância nos resultados de desempenho acadêmico e competência acadêmica, respectivamente. Esses resultados contrastam com o pequeno poder de predição dos indicadores de dificuldades adaptativas, com $\Delta \mathrm{R}^{2}$ entre $0,02 \mathrm{e}$ 0,05. Chama a atenção, em particular, a ausência do indicador de comportamentos externalizantes no 
modelo final preditivo, em desacordo com os achados de Burt e Roisman (2010). Vale ressalvar que esses autores trabalharam com crianças inicialmente mais jovens, frequentando o jardim de infância, e tiveram como informantes as mães e não os professores. Tais diferenças entre os estudos podem explicar em parte a divergência nos resultados.

Na presente investigação, para ambos os desfechos, o modelo final incluiu como preditores da criança as habilidades acadêmicas básicas, as habilidades sociais e a percepção de estressores escolares no $3^{\circ}$ ano. A presença desta última variável reitera a associação entre a percepção de stress na escola e um desempenho escolar mais fraco (Marturano et al., 2009). Em termos de causalidade, parece plausível supor um processo de retroalimentação, em que o desempenho pobre desencadeia pressões por parte dos adultos; estas, uma vez percebidas como estressores (Byrne et al., 2011), podem ocasionar problemas internalizantes (Leung et al., 2010), caracterizando um processo de internalização das dificuldades acadêmicas (Ackerman et al., 2007; Burt \& Roisman, 2010). Problemas internalizantes por sua vez interferem no progresso escolar, quando intensos (Grover et al., 2007).

Ao passo que recursos e dificuldades da criança contribuíram substancialmente para a predição dos dois desfechos investigados, as variáveis segregadas no primeiro bloco do modelo de regressão tiveram uma contribuição mais modesta. Gênero, NSE e atributos da escola de EF responderam, em conjunto, por $21 \%$ da variância nos resultados da prova objetiva de desempenho; para competência acadêmica, o percentual foi $4 \%$, explicado pelo gênero apenas. O tempo de exposição à EI, que nas comparações de grupo foi associado ao desempenho acadêmico no $5^{\circ}$ ano, não apareceu no modelo de predição quando em associação com as demais variáveis do ambiente. Esse resultado deve ser visto com cautela e requer verificação, dado que maior tempo na EI tem sido associado a melhor alfabetização, melhor desenvolvimento intelectual e maior sociabilidade (Felício, Terra, \& Zoghbi, 2012; Tagartt et al., 2011).

A escola de EF foi um importante preditor de desempenho acadêmico no $5^{\mathrm{O}}$ ano, mantendo-se no modelo mesmo depois da inclusão dos recursos acadêmicos e interpessoais da criança. Salles et al. (2010) já tinham constatado diferenças entre escolas públicas do mesmo município, ao investigarem o desempenho de alunos do EF em leitura e escrita. No entanto, com a inclusão das dificuldades adaptativas da criança no modelo de regressão, a escola de EF sai do modelo, um resultado que sugere efeito moderador das variáveis da criança nessa associação. Dado que neste estudo as escolas foram pontuadas de acordo com a origem social dos alunos, a configuração dos resultados obtidos sugere, por um lado, que a concentração de alunos em desvantagem socioeconômica é condição de vulnerabilidade para desempenho individual pobre (Aikens \& Barbarin, 2008), e, por outro, que esse efeito seria moderado por dificuldades adaptativas da criança, também influenciadas pelas características da escola, particularmente no que se refere ao comportamento (Rutter \& Maugham, 2002).

Na predição da competência acadêmica avaliada pelo professor, o nível atribuído às escolas de EF inicialmente não compõe o modelo; entretanto, passa a fazer parte dele em direção negativa quando as habilidades acadêmicas e sociais da criança são incluídas. Parece, então, que os recursos acadêmicos e interpessoais prévios do aluno têm maior peso sobre a avaliação de competência feita pelo professor, nas escolas com menos recursos.

Outra interpretação plausível para esse resultado inesperado pode ser formulada nos termos que seguem. As escolas com melhor pontuação socioeconômica são também aquelas que obtêm as melhoras notas no IDEB, o que pode estar associado a critérios mais exigentes de avaliação da competência do aluno. Dado que no instrumento utilizado para avaliação da competência acadêmica o professor avalia a posição da criança em relação aos colegas da classe, pode ter acontecido de os professores das escolas com melhor pontuação socioeconômica situarem em níveis mais extremos de competência os alunos percebidos como os mais fortes ou os mais fracos academicamente. $\mathrm{O}$ exame dos desvios-padrão na Tabela 2 mostra que, de fato, na escola Delta, com o melhor IDEB, houve maior dispersão na medida de competência acadêmica em relação à escola Beta, com o menor IDEB. Desse modo, alunos com resultados semelhantes no TDE ( $3^{\circ}$ ano) podem ter recebido pior avaliação de competência na escola Delta que na Beta (5ำ ano). Tal ocorrência explicaria o resultado à primeira vista contraintuitivo da análise de regressão.

Este estudo tem limitações que devem ser levadas em consideração na apreciação dos resultados. Uma delas diz respeito aos indicadores empregados para investigar a influência da escola, bastante restritos ante o complexo de fatores subjacentes ao chamado efeito escola, tais como recursos físicos e humanos, qualidade do ensino, características do professor, relacionamentos professor-aluno e estratégias de aproximação com as famílias (Pascual, 2011; Rutter \& Maugham, 2002; Salles et al., 2010). Outra limitação é a não inclusão de variáveis da família, reconhecidamente relevantes para o aprendizado escolar, como assinalado na 
introdução. Cabe lembrar ainda o caráter correlacional do delineamento, que não permite afirmar causalidade entre as variáveis estudadas, mas apenas precedência temporal.

Isso posto, a pesquisa contribui para a compreensão do curso de desenvolvimento acadêmico das crianças no primeiro ciclo do EF, seja pela confirmação de resultados de pesquisas prévias, seja pelo aporte de informação nova, tal como a que salienta as vivências de stress na escola como uma condição relevante na análise desse percurso. Como um esforço exploratório no sentido de integrar as informações esparsas disponíveis, conforme apontado na introdução, o estudo apresenta um modelo multivariado de predição de desempenho, assinalando a contribuição relativa de variáveis da criança e da escola. Nesse modelo, que se mostrou robusto na predição do desempenho no $5^{\mathrm{O}}$ ano, sobressaem as habilidades acadêmicas do aluno desenvolvidas previamente no contexto escolar, além da destacada primazia dos recursos sobre os indicadores de vulnerabilidade. A tentativa se mostrou frutífera ao sugerir processos por meio dos quais a influência da escola seria por assim dizer moderada pelas habilidades anteriormente desenvolvidas pelos alunos e por suas vivências prévias no espaço escolar. Desse modo, ainda que com participação direta discreta ou inexistente no modelo final de predição, a escola se apresenta com importante contribuição indireta, seja positiva, na construção das habilidades previas do aluno, seja negativa, por meio de experiências adversas percebidas pela criança como perturbadoras.
Assim, os achados aqui apresentados contribuem para o avanço do conhecimento na área ao fornecerem subsídio empírico para a construção de modelos teóricos de predição de desfechos acadêmicos no $5^{\circ}$ ano do ensino fundamental, a partir de características do aluno e da escola, avaliadas no $3^{\circ}$ ano. Um modelo fundamentado nos resultados da pesquisa deveria necessariamente privilegiar, entre os preditores, os recursos da criança em detrimento de suas fragilidades, bem como prever para a escola vias de influência tanto direta como indireta, positiva como negativa, intermediada por variáveis de competência e vulnerabilidade do aluno. Em decorrência de uma contribuição inédita deste estudo, tal modelo deveria ainda incluir entre os preditores de desfechos acadêmicos a visão da própria criança sobre situações adversas vividas no contexto escolar ou a ele diretamente relacionadas. Em pesquisas futuras, outros fatores podem ser agregados a esse plano básico de investigação, como, por exemplo, práticas parentais ou envolvimento da família na vida escolar da criança.

Como contribuição para práticas e políticas públicas, os resultados realçam a importância do investimento educacional no desenvolvimento das habilidades acadêmicas básicas e das habilidades sociais para promover o progresso escolar dos alunos. Eles também enfatizam a necessidade de que esse investimento se atualize em contexto de acolhimento, não ameaçador, de modo a minimizar os custos emocionais contraproducentes da exposição precoce a tensões cotidianas na escola.

\section{REFERÊNCIAS}

Ackerman, B. P., Izard, C. E., Kobak, R., Brown, E. D. \& Smith, C. (2007). Relation Between Reading Problems and Internalizing Behavior in School for Preadolescent Children From Economically Disadvantaged Families. Child Development, 78, 581-596. http://dx.doi.org/10.1111/j.1467-8624.2007.01015.x

Aikens, N. L., \& Barbarin, O. (2008). Socioeconomic differences in reading trajectories: The contribution of family, neighborhood, and school contexts. Journal of Educational Psychology, 100, 235-251. http://dx.doi.org/10.1037/00220663.100.2.235

Associação Brasileira de Empresas de Pesquisa. (2003). Critério de classificação econômica Brasil. Dados com base no levantamento sócio-econômico, IBOPE, 2000. Acessado em 14 fev. 2007, em http://www.abep.org.br

Bandeira, M., Del Prette, Z. A. P., Del Prette, A., Magalhães, T. (2009). Validação das escalas de habilidades sociais, comportamentos problemáticos e competência acadêmica (SSRS-BR) para o ensino fundamental. Psicologia: Teoria e Pesquisa, 25, 271-282. http://dx.doi.org/10.1590/S0102-37722009000200016

Burt, K. B., \& Roisman, G. I. (2010). Competence and psychopathology: cascade effects in the NICHD Study of Early Child Care and Youth Development. Development and Psychopathology, 22, 557-567. http://dx.doi.org/10.1017/ S0954579410000271

Byrne, D. G., Thomas, K. A., Burchell, J. L., Olive, L. S., \& Mirabito, N. S. (2011). Stressor Experience in Primary School-Aged Children: Development of a Scale to Assess Profiles of Exposure and Effects on Psychological WellBeing. International Journal of Stress Management, 18, 88-111. http://dx.doi.org/10.1037/a0021577

Caprara, V., Barbaranelli, C., Pastorelli, C., Bandura, A., \& Zimbardo, P. G. (2000). Prosocial foundations of children's academic achievement. Psychological Science, 11, 302-306. http://dx.doi.org/10.1111/1467-9280.00260 
Chen, X., Huang, X., Chang, L., Wang, L., \& Dan, L. (2010). Aggression, social competence, and academic achievement in Chinese children: a 5-year longitudinal study. Development and Psychopathology, 22, 583-592. http://dx.doi. org/10.1017/S0954579410000295

Escolano, A. C. M. (2004). Fatores de risco e proteção na trajetória de desenvolvimento de escolares de primeira à quarta série do ensino fundamental. Tese de doutorado não publicada. Universidade de São Paulo, Ribeirão Preto, SP.

Felício, F., Terra, R., \& Zoghbi, A. C. (2012). The Effects of Early Childhood Education on Literacy Scores Using Data from a New Brazilian Assessment Tool. Estudos Econômicos, 42, 97-128. http://dx.doi.org/10.1590/S010141612012000100004

Gardinal-Pizato, E. C. (2010). Um estudo longitudinal de trajetórias de desempenho escolar. Tese de doutorado não publicada. Ribeirão Preto, Universidade de São Paulo.

Grover, R. L., Ginsburg, G. S., \& Ialongo, N. (2007). Psychosocial outcomes of anxious first graders: A seven-year follow-up. Depression and Anxiety, 24, 410-420. http://dx.doi.org/10.1002/da.20241

Konold, T. R., Jamison, K. R., Stanton-Chapman, T.L., \& Rimm-Kaufman, S.E. (2010). RelationshipsAmong InformantBased Measures of Social Skills and Student Achievement: A Longitudinal Examination of Differential Effects by Sex. Applied Developmental Science, 14, 18-34. http://dx.doi.org/10.1080/ 10888690903510307

Leung, G. S. M., Yeung, K. C.\&. Wong, D. F. K. (2010). Academic Stressors and Anxiety in Children: The Role of Paternal Support. Journal of Child and Family Studies, 19, 90-100. http://dx.doi.org/10.1007/s10826-009-9288-4

Marturano, E. M., Trivelatto-Ferreira, M. C., Gardinal, E. C. (2009). Estresse cotidiano na transição da 1ª série: percepção dos alunos e associação com desempenho e ajustamento. Psicologia Reflexão e Crítica, 22, 93-101. http://dx.doi. org/10.1590/S0102-79722009000100013

Pascual, J. (2011). El efecto escuela. más allá del aula. Revista Iberoamericana sobre Calidad, Eficacia y Cambio en Educación, 9, 28-45. Acessado em: 24 mar. 2011, em http://www.rinace.net/reice/numeros/arts/vol9num1/art2.pdf

Romano, E., Babchishin, L., Pagani, L. S., \& Kohen, D. (2010). School Readiness and Later Achievement: Replication and Extension Using a Nationwide Canadian Survey. Developmental Psychology, 46, 995-1007. http://dx.doi. org/10.1037/a0018880

Rutter, M., \& Maugham, B. (2002). School Effectiveness Findings 1979-2002. Journal of School Psychology, 40, 451475. http://dx.doi.org/10.1016/S0022-4405(02)00124-3

Salles, J. F., Parente, M. A. M. P., \& Freitas, L. B. L. (2010). Leitura/escrita de crianças: comparações entre grupos de diferentes escolas públicas. Paidéia (Ribeirão Preto), 20(47), 335-344. http://dx.doi.org/10.1590/S0103$863 \mathrm{X} 2010000300006$

Stein, L. M. (1994). TDE - Teste de Desempenho Escolar: manual para aplicação e interpretação. São Paulo: Casa do Psicólogo.

Tagartt, B., Sylva, K., Melhuish, E., Sammons, P., \& Siraj-Blatchford, I. (2011). O poder da pré-escola: evidências de um estudo longitudinal na Inglaterra. Cadernos de Pesquisa, 41(142), 68-87. http://dx.doi.org/10.1590/S010015742011000100005

Nota:

Pesquisa realizada com apoio do CNPq e da FAPESP.

Autores:

EdNa MARIa Marturano - Doutora, Universidade de São Paulo, Faculdade de Medicina de Ribeirão Preto.

ElAiNE CRISTINA GARDINAL-PIZATO - Doutora, Fundação Educacional Dr. Raul Bauab, Faculdades Integradas de Jaú.

Endereço para correspondência:

Edna Maria Marturano

Faculdade de Medicina de Ribeirão Preto

Bloco da Saude Mental

Rua Tenente Catão Roxo, 2652

CEP 14051-140 Ribeirão Preto, SP, Brasil

Recebido em: 03.09.13

Aceito em: 15.08.14 